\title{
Los estilos de liderazgo en empresarias Ecuatorianas y su influencia en el desempeño de sus colaboradores
}

\section{The styles of leadership in Ecuadorian businesswomen and their influence on the performance of their collaborators}

María Auxiliadora Guerrero Bejarano

Universidad Internacional del Ecuador, Ecuador

Autor para correspondencia: anchavezer@internacional.edu.ec

Fecha de recepción: 01 de Febrero de 2016 - Fecha de aceptación: 25 de Febrero de 2017

\section{Resumen}

Si analizamos en el nivel empresarial, cuando pensamos en Líderes, nos referimos a aquellas personas que guían a las organizaciones dentro de los lineamientos que establecen para alcanzar los objetivos propuestos. Partiendo de esta idea, encontramos que la mujer va tomando un rol cada vez más importante en el desarrollo de las empresas, ejerciendo cargos gerenciales e involucrándose en la toma de decisiones y planificación estratégica de las mismas. Por sus habilidades intrínsecas, como la organización, suelen manejar eficientemente los recursos; esta misma creatividad con la que desempeñan sus tareas, les permite también generar nuevos negocios y convertirse en Empresarias. Este estudio permitirá examinar las diferencias de géneros que se pueden presentar en los estilos de liderazgo y su influencia en los equipos de trabajo, concretamente analizaremos las percepciones que los diferentes equipos de trabajo tienen respecto a los estilos de liderazgos de sus jefes.

Palabras claves: líderes; habilidades; estilos de liderazgos

\begin{abstract}
If we analyze at the business level, when we think of Leaders, we refer to those people who guide the organizations within the guidelines that they establish to reach the proposed objectives. Starting from this idea, we find that the woman is taking an increasingly important role in the development of companies, exercising managerial positions and getting involved in decision making and strategic planning of the same. Because of their intrinsic abilities, such as organization, they usually manage resources efficiently; this same creativity with which they perform their tasks, also allows them to generate new businesses and become Businesswomen. This study will allow us to examine the gender differences in leadership styles and their influence on the work teams, specifically analyzing the perceptions that the different teams have regarding the leadership styles of their bosses.
\end{abstract}

Key Words: leaders; skills; styles of leadership 


\section{Introducción}

Pensar en liderazgo siempre conduce a recordar líderes, personajes trascendentales como Nelson Mandela, Gandhi, Martín Luther King entre otros, quienes a través de sus acciones cambiaron la historia de su contexto en primer lugar, para luego convertirse en fuentes de inspiración y cambio globalmente. Las mujeres han ido desempeñando papeles importantes en el transcurso de la historia volviéndose generadoras de cambio, en muchos casos venciendo limitaciones y luchando contra factores discriminatorios, que la época y la cultura les imponían, encontramos ejemplos de liderazgo femenino en la Madre Teresa de Calcuta, Marie Curie, Juana de Arco, Manuela Sáenz y otras mujeres históricas que resultan fuentes de inspiración para líderes modernas como Michelle Bachelet.

Pero si lo analizamos en el nivel empresarial, cuando pensamos en Líderes, nos referimos a aquellas personas que guían a las organizaciones dentro de los lineamientos que establecen para alcanzar los objetivos propuestos. Partiendo de esta idea, encontramos que la mujer va tomando un rol cada vez más importante en el desarrollo de las empresas, ejerciendo cargos gerenciales e involucrándose en la toma de decisiones y planificación estratégica de las mismas. Por sus habilidades intrínsecas, como la organización, suelen manejar eficientemente los recursos; esta misma creatividad con la que desempeñan sus tareas, les permite también generar nuevos negocios y convertirse en Empresarias. Este estudio permitirá examinar las diferencias de géneros que se pueden presentar en los estilos de liderazgo y su influencia en los equipos de trabajo, concretamente analizaremos las percepciones que los diferentes equipos de trabajo tienen respecto a los estilos de liderazgos de sus jefes.

\section{Antecedentes}

Algunas investigaciones previas muestran que el estilo del liderazgo en las mujeres en se caracteriza por tener cualidades del liderazgo transformacional, al tener conductas sociales y emocionales que le permiten relacionarse de forma más cercana con sus colaboradores. Se hace una comparación entre el liderazgo masculino que se presume es más parecido al liderazgo militar contra el liderazgo femenino que es mucho más inspiracional. Entonces dados los efectos positivos del liderazgo carismático en las actitudes y rendimiento de los seguidores y la cohesión del grupo de trabajo, definen que las mujeres podrían tener una clara ventaja con respecto a las habilidades interpersonales y los comportamientos necesarios para un liderazgo efectivo, orientado al cambio organizacional (Koneck, Carolyn 2006).

Para Prentice, (1961) el desarrollo de sus empleados, para los líderes efectivos, en el largo plazo es un tema de interés personal, y usan el tacto y otras habilidades sociales para estimularlos a lograr lo mejor. Dista de ser "simpático" o "comprensivo", se trata de utilizar las motivaciones individuales en beneficio de promover un objetivo para toda la organización. Define el liderazgo como el logro de una meta mediante la dirección de colaboradores humanos, y al líder como la persona que organiza exitosamente a estos colaboradores para conseguir metas específicas. Afirma que el logro excepcional de un gran líder es de tipo social y humano y proviene de comprender a sus colaboradores y que una organización debe tener empleados en todos los niveles que reporten a alguien cuya área de autoridad tenga un tamaño adecuado para que pueda conocer a sus colaboradores en su dimensión humana. La tarea del 
líder es proporcionar la identificación de roles y funciones dentro del grupo que permita a cada miembro realizar un propósito o interés mayor.

La personalidad del gerente versus la del líder, Zaleznik, Abraham (1977), en un artículo en la revista Harvard Business Review define que los Gerentes y los Líderes tienen personalidades diferentes, debido a la naturaleza de las organizaciones, argumenta que una cultura gerencial enfatiza la racionalidad y el control, que el gerente es una persona que resuelve problemas, sus energías siempre se orientarán al cumplimiento de metas, manejo de recursos y control de la estructura de la organización o personas, siempre se preguntará:

“¿Cuáles son los problemas que hay que resolver y cuáles son las mejores formas de lograr resultados para que la gente siga contribuyendo a esta organización?". Desde esta perspectiva, el liderazgo es un esfuerzo que busca encaminar los asuntos; y cumplir con su cometido, un gerente necesita personas operando eficientemente en sus distintos niveles jerárquicos. Ser gerente no requiere genio o heroísmo, sino, perseverancia, determinación, trabajo duro, inteligencia, capacidad analítica, y quizás lo más importante: tolerancia y buena voluntad. Otra visión de liderazgo, supone que sólo las personas extraordinarias merecen participar del poder y la política. Esta expectativa del liderazgo contrasta fuertemente con la concepción cotidiana, práctica, pero importante, que asegura que el liderazgo es realmente administrar el trabajo de los demás. Gerentes y líderes son personas muy distintas. Difieren en cuanto a su motivación, su historia personal, y también, en su modo de pensar y actuar.

Goleman, Daniel (2011) afirmó que aunque el coeficiente intelectual y las destrezas técnicas son importantes en el desarrollo de un líder, es la inteligencia emocional la que realmente hace la diferencia en ser o no uno. También explica que los estilos personales de los líderes sobresalientes varían: siendo algunos moderados y analíticos, mientras que otros son más expresivos a la hora de exponer su ideas; pese a estas diferencias encuentra aspectos parecidos en los líderes más efectivos, todos tienen un alto grado de inteligencia emocional, sin ella una persona pese a tener la mejor preparación del mundo, una capacidad analítica envidiable, podría tener problemas para liderar un grupo de trabajo. Encuentra que es la variable que diferencia a un líder de alto nivel, de uno de nivel intermedio, debido a que a medida que se avanza en una jerarquía las habilidades técnicas distan de ser muy diferentes, todo está en el poder controlar de mejor forma los impulsos y emociones. Se habla de una madurez emocional que permite generar empatía con su equipo y mantener un equilibrio para el desarrollo de las actividades del mismo.

\section{Declaración del Problema}

Este estudio pretende analizar las supuestas diferencias en los estilos de liderazgo de hombres y mujeres. Así como si dichos estilos afectan el desempeño de los grupos que dirigen. Si los resultados de los estudios realizados en países como Estados Unidos e Inglaterra pueden ser replicados en países de Latinoamérica como Ecuador.

\section{Propósito de la Investigación}


El propósito de este estudio cuantitativo es comprobar las posibles diferencias entre los distintos estilos de liderazgo presentes en los hombres y mujeres, así como la influencia de éstos en el desarrollo de los grupos que dirigen.

\section{Importancia del Estudio}

Al realizar este estudio podremos comprender de mejor forma a las líderes femeninas que se desarrollan en nuestro tiempo, y si existe diferencia entre los estilos de liderazgo entre los géneros y si este estilo permite que los grupos de trabajo se desempeñen de mejor manera.

\section{Naturaleza del Estudio}

Este estudio será desarrollado bajo la metodología cuantitativa, se realizarán encuestas utilizando el cuestionario multifactorial de liderazgo (MLQ) desarrollado por B. Bass y B. Avolio, aplicándolo en equipos de trabajo de líderes hombres y mujeres de diferentes empresas de la ciudad de Guayaquil. De esta forma podremos confirmar si el estilo de liderazgo que predomina en las mujeres es el transformacional y si existe una diferencia marcada en el estilo del liderazgo a causa del género.

\section{Preguntas de Investigación}

$\checkmark$ ¿Cuáles son las habilidades de liderazgo de las mujeres?

$\checkmark$ ¿Cuáles son las diferencias entre los estilos de liderazgo entre los hombres y las mujeres?

$\checkmark \quad$ ¿Tiene el estilo de liderazgo de los gerentes influencia en el desempeño de sus colaboradores?

\section{Hipótesis}

$\checkmark$ H1: En general el estilo de liderazgo que predomina en las mujeres es el transformacional

$\checkmark$ H2: Los hombres utilizan en general el estilo de liderazgo transaccional para guiar a sus grupos de trabajo

$\checkmark$ H3: El estilo de liderazgo de los líderes influye directamente en el desempeño de sus grupos de trabajo

\section{Marco Conceptual}

Liderazgo es un proceso que involucra una visión y un pensamiento de largo plazo implícito, donde la transformación y el cambio a ser mejores es inherente. Sobre todo, en la transformación de las organizaciones y sus culturas. La gerencia es algo de más corto plazo y operacional. En el liderazgo cómo y en la gerencia se consiguen resultados, con la diferencia de hacerlo en el largo o en el corto plazo respectivamente. En el liderazgo existe una acción voluntaria de los seguidores, quienes actúan por el carisma, personalidad, y credibilidad del líder. (DÁlessio, 2009)

Liderazgo Transaccional se refiere al intercambio entre el líder y sus seguidores con la finalidad de que sus intereses coincidan, mediante recompensas o castigos; generalmente el líder junto con el seguidor define los requisitos que se deben cumplir para poder ser recompensado. 
En este enfoque se puede identificar las necesidades de los subordinados para así poder emplear con eficiencia los objetivos institucionales al generar tareas. (Beraún Quiñones, Lida 2011)

Liderazgo Transformacional: en esta teoría se toma en cuenta los rasgos y conductas del líder, así como las variables situacionales; cobran especial importancia las actitudes y percepciones que sostienen los seguidores respecto a sus líderes. Los seguidores no solo confían y respetan a su líder sino que lo idealizan como una figura excepcional y valoran características como sus convicciones, autoconfianza y su anhelo de poder. (Beraún Quiñones, Lida 2011)

\section{Variables independientes}

Los Estilos de Liderazgo mencionados anteriormente (a) Transaccional y (b) Transformacional son las variables independientes en este estudio, que busca analizar si realmente afectan el desempeño de los equipos de trabajo

Liderazgo es un proceso que involucra una visión y un pensamiento de largo plazo implícito, donde la transformación y el cambio a ser mejores es inherente. Sobre todo, en la transformación de las organizaciones y sus culturas. La gerencia es algo de más corto plazo y operacional. En el liderazgo cómo y en la gerencia se consiguen resultados, con la diferencia de hacerlo en el largo o en el corto plazo respectivamente. En el liderazgo existe una acción voluntaria de los seguidores, quienes actúan por el carisma, personalidad, y credibilidad del líder. (DÁlessio, F.A. 2009)

\section{Estilos de Liderazgo}

Diferentes estudios definen que los líderes tienen como función crear un clima o atmósfera social en el grupo que influirá la satisfacción y rendimientos de sus miembros. En estos estudios se realizaron manipulaciones experimentales para comprobar tres tipos de estilos diferentes de liderazgo: el autocrático, cuando el líder organizaba todas las actividades del grupo, daba las órdenes, dando las instrucciones de lo que cada uno debería y no hacer. El líder democrático, cuando fomentaba la participación de los miembros del grupo a la hora de tomar decisiones. Finalmente el liderazgo laissez faire, en dónde el líder adoptaba un comportamiento pasivo, no tomaba iniciativas, tampoco juzgaba o evaluaba ningún tipo decisión. (Cuadrado, I, 2001)

Liderazgo Transaccional se refiere al intercambio entre el líder y sus seguidores con la finalidad de que sus intereses coincidan, mediante recompensas o castigos; generalmente el líder junto con el seguidor define los requisitos que se deben cumplir para poder ser recompensado. En este enfoque se puede identificar las necesidades de los subordinados para así poder emplear con eficiencia los objetivos institucionales al generar tareas. (Beraún Quiñones, Lida 2011). El liderazgo Transaccional está formado por dos factores, el primero es la capacidad del líder de recompensar a los miembros de su equipo por la labor bien hecha, recompensa contingente. El segundo factor es la dirección por excepción que es cuando los líderes intervienen de manera reactiva, solamente para reprender o castigar a sus subordinados. (Bass y Avolio, 1990) 
Liderazgo Transformacional: en esta teoría se toma en cuenta los rasgos y conductas del líder, así como las variables situacionales; cobran especial importancia las actitudes y percepciones que sostienen los seguidores respecto a sus líderes. Los seguidores no solo confían y respetan a su líder sino que lo idealizan como una figura excepcional y valoran características como sus convicciones, autoconfianza y su anhelo de poder. (Beraún Quiñones, Lida 2011).

Básicamente el liderazgo transformacional está formado por cuatro factores o dimensiones: carisma o influencia idealizada, que es la capacidad del líder de evocar una visión y lograr la confianza de su equipo de trabajo. El segundo factor es la inspiración o motivación inspiracional que es la capacidad del líder de comunicar su visión. La estimulación intelectual como tercer factor, es la capacidad del líder de hacer que sus subordinados piensen de manera creativa o innovadora, de manera participativa. Finalmente la consideración individualizada que consiste en la capacidad del líder de prestar atención personal a todos los miembros de su equipo, haciendo que cada uno sienta el valor que su función tiene en el desempeño del conjunto. (Bass y Avolio, 1990)

\section{Variables moderadoras}

La cultura nacional, el tamaño de la organización y el género son las variables moderadoras del estudio, nos ayudarán a determinar si existe una diferencia en los resultados si los equipos de trabajo son distintos debido a las posibles variaciones en el tamaño de la organización, género de los líderes y la cultura nacional del país, en este caso Ecuador. Sobre la cultura nacional, basados en la propuesta de Hofstede (1991) se consideran las dimensiones de distancia de poder, individualismo, masculinidad y aversión a la incertidumbre. A pesar de que la muestra será diferenciada por sectores industriales, no se considera esta diferencia una variable moderadora en este estudio. El tamaño de la organización por otro lado sí, pues en estudios previos fue considerado un factor que será medido por el número de trabajadores que poseen, considerando empresas pequeñas, medianas y grandes.

\section{Variable Dependiente}

El desempeño, es la única variable dependiente del estudio y será medido por los resultados obtenidos por los distintos equipos de trabajo. Será medido el desempeño operacional considerando la satisfacción del cliente, el nivel de calidad y la reputación de la empresa.

\section{Definiciones}

Líder Corporativo: persona que guía, orienta, motiva e influye a un grupo de personas para conseguir los objetivos trazados dentro de una empresa, basado en los aspectos definidos en la cultura organizacional.

Cultura Organizacional: los valores, principios y tradiciones que predominan en el ambiente profesional de una organización, esta cultura se manifiesta en la conducta y en la forma de comunicarse de las personas. 
Estereotipo: conceptos establecidos en la mente de las personas que generalmente rigen su patrón de conducta.

Aspiración: pretensión o meta que busca obtener un individuo, muchas veces la aspiración o aspiraciones se convierten en la razón o motivación que permite que la persona se desarrolle en algún campo específico.

Carisma: capacidad de ciertas personas para motivar e influir en el comportamiento de los demás

Nivel Intermedio: cargos de mediana responsabilidad, se caracterizan por tener que supervisar el trabajo de otras personas y al mismo tiempo deben rendir cuentas a un nivel superior.

Alto Nivel Jerárquico: cargos gerenciales de primer nivel, como gerencias generales, en estas posiciones las personas se ocupan de actividades como la planificación estratégica de la organización que dirigen.

Inteligencia Emocional: capacidad de los individuos para controlar los sentimientos, impulsos y estímulos a nivel cerebral, permitiéndole ser empático con las otras personas teniendo autoconsciencia de sus propios límites y capacidades.

Cultura: factores externos que influyen en el comportamiento de las personas, dichos aspectos están relacionados con el entorno en el que las personas se desarrollan y pueden generar diferencias sustanciales en el establecimiento con los demás individuos.

\section{Asunciones}

Para Koontz, Harold; Weihrich, Heinz y Cannice, Mark (2008) el liderazgo es el arte o proceso de influir en las personas para que se esfuercen voluntaria y entusiastamente en el cumplimiento de metas grupales. Chiavenato, Idalberto (1993), define el liderazgo como una influencia interpersonal ejercida en una situación y dirigida a través de un proceso de comunicación humana para la consecución de objetivos específicos.

Koontz, Harold; et al (2008) definen como los componentes del Liderazgo: Capacidad para hacer un uso eficaz y responsable del poder; capacidad para comprender que los seres humanos tienen diferentes motivaciones en diferentes momentos y situaciones; capacidad para inspirar a los demás; capacidad para actuar a favor del desarrollo de una atmósfera conducente ante las motivaciones y al surgimiento de estas. El principio del liderazgo para Koontz, et al es que los individuos tienden a seguir a quienes, en su opinión, les ofrecen los medios para satisfacer sus metas personales. Por ello, cuanto mayor sea la comprensión de los administradores de lo que motiva a sus subordinados y de la forma como operan estas motivaciones, y cuánto más demuestren comprenderlo en sus acciones administrativas, tanto más eficaces serán probablemente como líderes. 
Los enfoques de los rasgos del liderazgo están definidos por los rasgos físicos (energía, apariencia y altura); los rasgos de inteligencia y capacidad; rasgos de personalidad como la adaptabilidad, agresividad, entusiasmo y seguridad en si mismo; características relativas al desempeño de tareas como el impulso de realización, persistencia e iniciativa; y las características sociales como el sentido de cooperación, habilidades para las relaciones interpersonales y capacidad administrativa. Rasgos claves del Liderazgo: impulso (anhelo de realización, motivación, energía, ambición, iniciativa y tenacidad); motivación para el liderazgo (la aspiración a dirigir, aunque no a buscar el poder como tal); Honestidad e integridad; Seguridad en uno mismo (incluida estabilidad emocional); Capacidad cognoscitiva; Comprensión de los negocios y en menor proporción: creatividad, flexibilidad y carisma. Factores que contribuyen a un liderazgo eficaz: las características de los subordinados, como sus necesidades, grado de seguridad en sí mismos y capacidades y las condiciones de trabajo, incluidos componentes tales como tareas, sistema de recompensas y relaciones con los compañeros de trabajo. (Koontz, et al, 2008)

La Conducta del Líder: (a) liderazgo de apoyo, se toman en consideración las necesidades de los subordinados, se muestra interés por su bienestar y se crea un ambiente organizacional agradable. Esto ejerce el mayor impacto sobre el desempeño de los subordinados en caso de que se sientan frustrados o insatisfechos; (b) el liderazgo participativo permite a los subordinados influir en las decisiones de sus superiores y puede resultar en mayor motivación; (c) el liderazgo instrumental ofrece a los subordinados orientación más bien específica y aclara lo que se espera de ellos; incluye aspectos de planeación, organización, coordinación y control por parte del líder, (d) el liderazgo orientado a logros implica el establecimiento de metas ambiciosas, la búsqueda de mejoras del desempeño y la seguridad en que los subordinados alcanzarán metas elevadas. (Koontz, et al, 2008)

\section{Limitaciones}

Se asume que las respuestas que se obtendrán de las personas que participen en la investigación serán otorgadas en base a la verdad y a la buena voluntad de los mismos. Se asume que el desempeño es mayormente afectado por el estilo de liderazgo. Se aplicará la herramienta en un solo momento del tiempo, por lo que no se podrá luego observar si se presentarían resultados diferentes a los que se obtendrán al hacer variaciones en las variables. Los datos que se obtendrán serán subjetivos al ser las opiniones de los encuestados, por lo que existirá un riesgo de sesgo en las respuestas dadas por los participantes, por esta razón se considerará trabajar con 40 trabajadores por empresa para reducir dicho riesgo. Se trabajará con una muestra de conveniencia y no probabilística, al encuestar a personas que trabajen en los departamentos de atención al cliente de las diferentes empresas escogidas a las que se tendrán acceso por relaciones.

\section{Delimitaciones}

Se delimita esta investigación a grupos de trabajo de diferentes empresas de la ciudad de Guayaquil, buscando representatividad con organizaciones medianas y grandes de sectores como: servicios bancarios, hotelería, entretenimiento, construcción, educación básica, educación superior y retailing. No se considerarán empresas del sector público, y se trabajará con empresas 
del sector de servicios principalmente. Las empresas escogidas para la muestra son medianas y grandes, según el INEC económico, 2011 las empresas medianas son las que tienen entre 10 y 199 empleados y las grandes son las que cuentan con más de 199 empleados, siendo las pequeñas las que tienen menos de 9 empleados, por esta razón al querer disminuir el riesgo del sesgo en las respuestas, no se tomarán en cuenta las empresas pequeñas en el desarrollo del estudio. Se trabajará con una muestra de conveniencia y no probabilística.

\section{Diseño de la Investigación}

Al tener este estudio como objetivo principal encontrar las posibles causas de diferentes resultados obtenidos, este estudio aplicará los paradigmas post positivistas para encontrar el grado de relación que pudiera encontrarse entre los estilos de liderazgo y el desempeño de los grupos de trabajo. Este estudio utilizará el método cuantitativo, trabajando con encuestas para la obtención de datos. Las encuestas buscarán confirmar que existe una relación directa entre las variables, razón por la que la investigación será confirmatoria. Para realizar la investigación se utilizará el cuestionario multifactorial de liderazgo, MLQ un instrumento diseñado por B. Bass y B. Avolio con el objetivo de medir los estilos de liderazgo definidos en el capítulo 2 del presente estudio, este instrumento permitirá obtener los resultados sobre los distintos estilos de liderazgo aplicados por los diferentes jefes de grupos y establecer si existe diferencias entre los líderes de acuerdo a su género. Para obtener los datos que nos permitirán conocer resultados de desempeño, se trabajará con una encuesta de medición de la satisfacción del cliente.

\section{Conveniencia del Diseño}

Este estudio busca confirmar la posible relación entre el estilo de liderazgo aplicado por los jefes de grupo y el desempeño de los mismos, así como las posibles diferencias en los estilos de liderazgo de acuerdo al género de los líderes, por este motivo este es un estudio de correlación. Existen cerca de una docena de estudios previos sobre la diferencia de género en los estilos de liderazgo transformacional y transaccional (Cuadrado I, Molero F y Navas M, 2003) en los que se utiliza el método cuantitativo para el análisis y el cuestionario MLQ como instrumento para la obtención de los resultados. Algunos evaluaron únicamente a los líderes, otros únicamente a los subordinados y en algunos casos a líderes y subordinados; en este caso se encuestarán a los subordinados para obtener los resultados sobre los estilos de liderazgo y a los clientes para obtener resultados sobre el desempeño.

\section{Característica de la Muestra}

Se determina una muestra de 40 trabajadores por empresa, 20 dirigidos por hombres y 20 dirigidos por mujeres, buscando representatividad con organizaciones medianas y grandes de sectores como: servicios bancarios, hotelería, entretenimiento, construcción, educación básica, educación superior y retailing. En total se trabajarán siete empresas una por sector, lo que nos permitirá tener una muestra de 280 personas de diferentes equipos de trabajo, 140 dirigidos por hombres y 140 dirigidos por mujeres. Se trabajará el estudio con personas de mandos medios y servicio al cliente, suponiendo que al tener contacto con el cliente, se podrá obtener información de los resultados de su desempeño con mayor facilidad al encuestar clientes sobre el nivel de satisfacción de los servicios recibidos. 


\section{Confidencialidad}

No se guardarán registro de las personas encuestadas para garantizar la confidencialidad de los datos y disminuir posibles sesgos en las respuestas, así como que los participantes puedan sentir algún tipo de temor al participar en el estudio.

\section{Localización Geográfica}

Se realizará el estudio en la ciudad de Guayaquil, en empresas medianas y grandes del sector de servicios, dedicadas a servicios bancarios, hotelería, entretenimiento, educación básica, educación superior, construcción y retailing.

\section{Instrumentación}

Se analizarán las diferentes variables que cada estilo de liderazgo tiene de acuerdo a las definiciones encontradas y revisadas en la literatura analizada, se utilizará un instrumento comprobado y validado en varios estudios previos, el cuestionario multifactorial de liderazgo creado con el objetivo de medir las diferencias entre los distintos estilos de liderazgo y la ausencia del liderazgo en un equipo de trabajo. La estructura factorial del MLQ -5X, consta de tres tipos de factores que miden cada uno de los estilos de liderazgos: transaccional, transformacional y Laissez-faire. Este instrumento está fundamentado de forma teórica en las ideas de Bass, quién defiende la existencia de dos tipos de liderazgo: el transaccional y el transformacional. El liderazgo transaccional, basado en el intercambio de recompensas entre el líder y los miembros de su equipo. Los empleados realizan su labor y a cambio el líder o directivo les proporciona recompensas económicas u otro tipo de refuerzos. Este tipo de liderazgo, puede conseguir efectos positivos en la satisfacción y rendimiento de los empleados si se aplica correctamente. (Molero, F; Recio, P y Cuadrado, I 2010)

El estudio del liderazgo transformacional no es nuevo, bajo el nombre de liderazgo carismático, se venía estudiando desde la sociología y la ciencia política desde que Max Weber acuñara este concepto. Bass (1985) tiene el mérito de haber cambiado esta concepción asumiendo que el liderazgo carismático (al que llama liderazgo transformacional) se produce en todos los ámbitos de la sociedad y con una relativa frecuencia. Por ello, probablemente, la aportación más importante de Bass y sus colaboradores es haber plasmado su concepción teórica en un cuestionario, el MLQ, capaz de medir el liderazgo transformacional. Con ello comienza uno de los paradigmas del liderazgo más importantes de los últimos años. (Molero, $\mathrm{F}$ y otros 2010)

\section{Bibliografía}

Bass, B.M. (1998) Transformational and transactional leadership of men and women. En B.M.

Bass, Transformational Leadership: Industrial, Military and Educational Impact (pp. 72-79). Nueva Jersey: Lawrence Erlbaum Associates

Bass, B.M. y Avolio, B.J. (1990). Transformational leadership development: Manual for the Multifactor Leadership Questionnaire. Palo Alto: Consulting Psychologist Press.

Bass, B.M. y Avolio, B.J. (1994) Shatter the glass ceiling: women may make better managers. Human Resource Management, 33 (4), 549-560. 
Bass, B.M., Avolio, B.J. y Atwater, L. (1996) the transformational and transactional leadership of men and women. Applied Psychology: An International Review, 45 (1), 5-34

Beraún Quiñones, L (2011) El liderazgo para generar capacidades, Cayhuana Pilcomarca, Perú: Mercurio Publicaciones

Carless, S.A. (1998). Gender differences in transformational leadership: An examination of superior, leader, and subordinate perspective. Sex Roles, 39, 887-902

Cuadrado, I. (2001). Cuestiones teóricas y datos preliminares sobre tres estilos de liderazgo. Revista de Psicología Social, 16 (2), 131-155

Cuadrado, I. y Molero F. (2002). Liderazgo Transformacional y género: autoevaluaciones de directivos y directivas españoles. Revista de Psicología Del Trabajo y de las Organizaciones 18(1), 3955.

Cuadrado, I, Molero, F. y Navas, M. (2003) El Liderazgo de hombres y mujeres: diferencias en estilos de liderazgo, relaciones entre estilos y predictores de variables de resultado organizacional. Revista Acción Psicológica 2(2), 115-129

Chiavenato, I (1993) Administración de recursos humanos. México: Mc Graw-Hill

D’Alessio, F. (2009), Liderazgo y atributos gerenciales. Una visión global y estratégica. México D.F., México: Pearson George, Bill; Sims, Peter; Mclean, Andrew y Mayer, Diana (2011) Descubra su auténtico liderazgo. Harvard Business Review 89(11), 10-17

Goleman, Daniel (2011) ¿Qué hace a un líder? Harvard Business Review, 89(11), 42-50

Hofstede, G. (1991). Cultures and organizations, software of the mind [Culturas y Organizaciones, software de la mente]. Londres: McGraw Hill.

Koneck, Carolyn M (2206) A Study of women leadership Styles and The Glass Ceiling. (Tesis Doctoral) Disponible en la base de tatos ProQuest Information and Learning Company. (UMI Number: 3239058)

Koontz, Harold; Weihrich, Heinz y Cannice, Mark (2008); Liderazgo (13ra Ed.) Administración: una perspectiva global y empresarial. México: McGraw-Hill

Molero, Fernando; Recio, Patricia y Cuadrado, Isabel (2010) Liderazgo transformacional y liderazgo transaccional: un análisis de la estructura factorial del Multifactor Leadership Questionnaire (MLQ) en una muestra española. Psicothema 22(3) 495-501

Prentice, W (1961), Comprendiendo el liderazgo. Harvard Business Review, Serie Liderazgo Lo que hacen los grandes líderes

Zaleznik, Abraham (1977), Gerentes y líderes: ¿son diferentes? Harvard Business Review, Serie Liderazgo Lo que hacen los grandes líderes 